\title{
Journee de dialogue sur la contraception d'urgence (CU)
}

\section{Population Council}

International Consortium for Emergency Contraception

Follow this and additional works at: https://knowledgecommons.popcouncil.org/departments_sbsr-rh

Part of the Demography, Population, and Ecology Commons, Family, Life Course, and Society Commons, International Public Health Commons, and the Women's Health Commons How does access to this work benefit you? Let us know!

\section{Recommended Citation}

"Journee de dialogue sur la contraception d'urgence (CU)," Meeting report. Dakar: Population Council, 2013. 


\section{(2) Population Council}

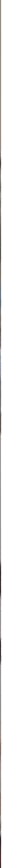

\section{JOURNEE DE DIALOGUE SUR LA CONTRACEPTION D'URGENCE (CU)}




\section{Table des matières}

Sigles et Acronymes ………………............................................................................................... ii

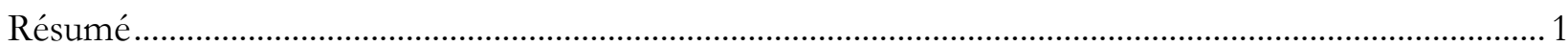

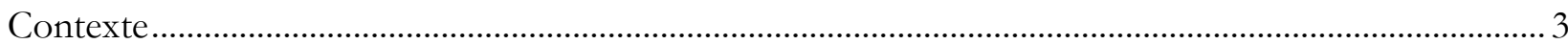

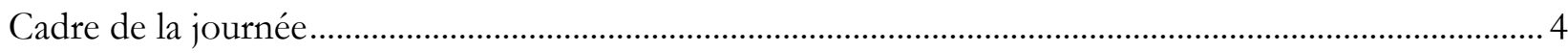

Objectifs de la journée de dialogue ....................................................................................................... 4

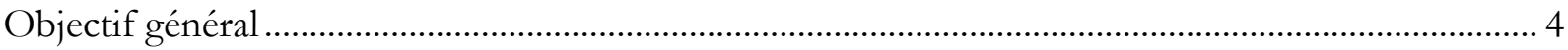

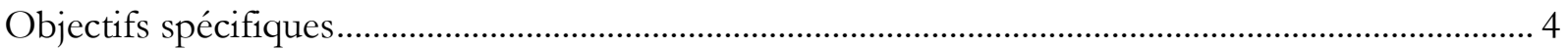

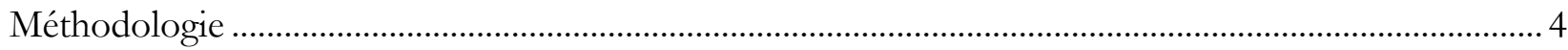

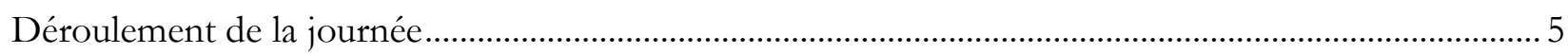

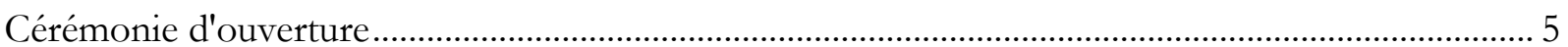

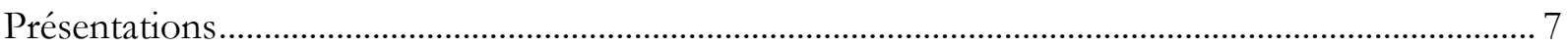

Partage des expériences des acteurs locaux ...................................................................................... 11

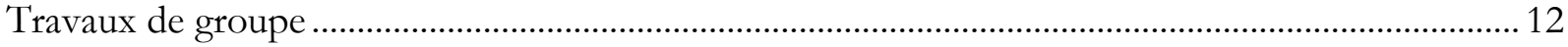

Déclaration conjointe d'engagement des trois Ministères partenaires ................................................ 14

Clôture de la journée de partage …………………………....................................................................... 15

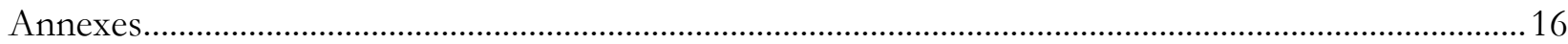

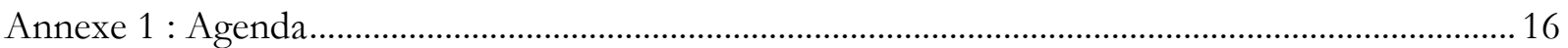

Annexe 2 : Travaux de groupe pour développer un plan d'action pour la vulgarisation de la

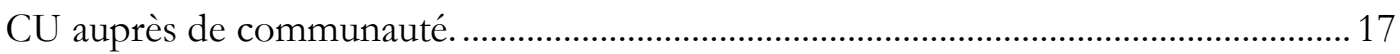

Annexe 3 : Draft - Déclaration conjointe d'engagement ......................................................................... 20

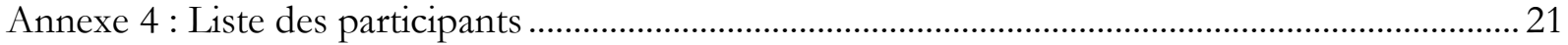




\section{SIGLES ET ACRONYMES}

ASBEF Association pour le Bien-être Familial

BNS Besoin Non Satisfait

CCA Centres Conseils Adolescents

CPRS Centre de Promotion et de Réinsertion Sociale

CU Contraception d'Urgence

DF Direction de la Famille

DPM Direction de la Pharmacie et du Médicament

DSRSE Direction de la Santé de la Reproduction et de la Survie de l'Enfant

EPS Education Pour la Santé

FAWE Forum for African Woman Educationalist

GEEP Groupe pour l'Enseignement et l'Education de la Population

GND Grossesse Non Désirée

ICEC Consortium International sur la Contraception d'Urgence

IST Infection Sexuellement Transmissible

MAC Maison d'Arrêt et de Correction

MFEEF Ministère de la Femme, de l'Enfant et de l'Entreprenariat Féminin

MSAS Ministère de la Santé et de l'Action Sociale

NTIC Nouvelles Techniques de l'Information et de la Communication

OCB Organisation Communautaire de Base

OMD Objectif du Millénaire pour Développement

PC Population Council

PF Planification Familiale

PNP Politiques, Normes et Protocoles

PPJ Projet Promotion des Jeunes

PPS Points de Prestations de Services

SR Santé de la Reproduction

SR/PF Santé de la Reproduction et Planification Familiale 


\section{RESUME}

Le 11 juin 2013, le Population Council en collaboration avec le Consortium International sur la Contraception d'Urgence (ICEC), a organisé, une journée de dialogue sur la Contraception d'Urgence (CU), en partenariat avec le Ministère de la Femme, de l'Enfant et de l'Entreprenariat Féminin, le Ministère de la Santé et de l'Action Sociale et le Ministère de la Jeunesse, de l'Emploi et de la Promotion des Valeurs Civiques.

\section{Objectif général}

L'objectif principal de la journée était de développer un plan d'actions pour la création d'un environnement politique et juridique plus favorable à l'offre de CU, et pour la vulgarisation de la CU auprès des communautés.

\section{Objectifs spécifiques}

Les objectifs spécifiques de la journée étaient de :

- Procéder à la mise à jour des connaissances sur la Contraception d'Urgence, les politiques et programmes existants au Sénégal ;

- Partager les résultats de recherche sur la CU faite par le Population Council et d'autres institutions ;

- Partager les expériences des institutions d'encadrement et de prise en charge des jeunes et des femmes en matière de santé sexuelle et reproductive et droits humains ;

- Identifier les contraintes sur le plan politico-juridique et proposer des solutions ;

- Informer le personnel de santé et les communautés sur la CU ;

- Développer un plan d'actions et discuter de sa mise en œuvre.

\section{Méthodologie}

La journée a été organisée en séances plénières et en travaux de groupe. Les travaux de groupe ont porté sur les trois thématiques suivantes :

- Plaidoyer pour une amélioration de l'environnement politique et juridique pour une meilleure accessibilité des services de CU;

- Vulgarisation de la CU et création de la demande;

- Offre de services de CU et intégration de la CU dans les structures formelles et non formelles.

Un modérateur a été recruté pour faciliter le déroulement des travaux.

\section{Principaux résultats}

La journée a permis de procéder à :

- La mise à jour des participants sur les dernières connaissances sur la CU (mode d'action, délai d'utilisation, utilisation répétée, agenda international pour améliorer l'accès à la CU...)

- Au partage des principaux résultats des recherches menées sur le sujet, notamment que: 
o Il existe une volonté effective des autorités publiques d'adresser la question des Grossesses Non Désirées (GND) et leurs corollaires (infanticides et avortements). Cette volonté s'est traduite par l'intégration depuis 2000 de la CU dans les documents officiels du Ministère de la Santé et de l'Action Sociale (PNP, curricula de formation...). Toutefois, la $\mathrm{CU}$ demeure encore peu connue des populations. L'attitude négative des prestataires combinée aux préjugés qui entourent le produit freinent sa promotion.

o La CU est un produit sûr et efficace si elle est utilisée dans de bonnes conditions. Son utilisation répétée n'a pas de conséquence majeure sur la santé de la femme, et les femmes peuvent l'utiliser sans danger plusieurs fois dans le même cycle menstruel. Toutefois l'utilisation de la CU en tant que méthode régulière de contraception n'est pas indiquée. D'autres méthodes plus efficaces de contraception sont disponibles.

o La promotion de la CU devra nécessairement passer par :

- La vulgarisation du produit auprès des populations à travers des campagnes de communication ciblant les Organisations Communautaires de Base (OCB), les membres de la société civile, les organisations/ institutions d'encadrement des femmes et des jeunes et les parlementaires;

- Le plaidoyer pour un assouplissement de l'environnement juridique et réglementaire plus favorable à l'offre de $\mathrm{CU}$;

- L'amélioration de l'offre par le biais de la formation des prestataires ;

- La création de la demande.

- Au partage des expériences des institutions d'encadrement et de prise en charge des jeunes et des femmes en matière de santé, notamment l'ASBEF, le Camp Pénal qui encadrent les femmes en milieu carcéral, la Direction de la Famille qui encadrent les associations et groupements de promotion des femmes.

- La revue des contraintes liées à l'offre et à l'accès aux services de CU.

- L'élaboration d'une esquisse de plan d'action pour la vulgarisation et la promotion de la CU. 


\section{CONTEXTE}

Depuis plus d'une décennie, de nombreux pays africains sont en train d'intégrer la Contraception d'Urgence (CU) dans leur programme de santé de la reproduction. Cette option s'inscrit dans la volonté des autorités publiques d'assurer une prévention efficace des Grossesses Non Désirées (GND) et de leurs conséquences que sont les avortements et les infanticides. La CU, en tant que méthode d'urgence, constitue un moyen très efficace de prévention de ces GND.

Le Ministère de la Santé et de l'Action Sociale (MSAS), avec l'appui du Population Council, a engagé par le biais de la Direction de la Santé de la Reproduction et de la Survie de l'Enfant (DSRSE), des initiatives soutenues de vulgarisation de la CU dans le Programme National de Planification Familiale (PNPF). Ainsi, depuis 2000, l'offre de la CU est intégrée dans les documents de Politiques, Normes et Protocoles (PNP) des services de Santé de la Reproduction et de Planification Familiale (SR/PF). La CU est ainsi inclue dans la formation en technologie contraceptive dispensée aussi bien lors de la formation de base que lors des formations en cours d'emploi des prestataires de services de SR.

Toutefois, plusieurs études menées récemment sur la CU au Sénégal, ont montré que, bien que la CU soit largement disponible au niveau des structures, elle n'est pas suffisamment vulgarisée dans le système de santé publique ainsi qu'au niveau des communautés. Une étude réalisée en 2011 par le Population Council sur 'les croyances, attitudes et pratiques des leaders d'opinion clés et des prestataires sur la Contraception d'Urgence" a mis en exergue cette faible connaissance des populations sur la CU. Le secteur privé, particulièrement les pharmacies privées, demeure la principale source d'approvisionnement de la CU. Plusieurs problèmes ont été répertoriés, notamment l'insuffisance voire l'absence d'informations des femmes sur la CU qui n'est pas inclue lors du counseling initial sur la PF, d'où la faible prescription/ provision de la méthode par les prestataires qui posent certaines restrictions. Il s'y ajoute le manque de connaissance spécifique des prestataires et autres acteurs clés sur le produit, notamment sur son mode d'action. Enfin, l'absence de statistiques fiables et exhaustives sur la distribution de la CU au niveau des pharmacies privées ne permet pas d'apprécier de façon fiable le niveau d'utilisation du produit et le nombre d'utilisateurs potentiels.

Le forum national sur la CU (octobre 2010) et l'atelier national de dissémination des résultats de l'étude sur les croyances, attitudes et pratiques des leaders d'opinion clés et des prestataires (novembre 2011) ont été des étapes clés qui ont permis de relancer le débat sur la CU. Ces deux réunions stratégiques avaient fortement recommandé d'accorder une priorité au renforcement de la connaissance de la CU chez les prestataires mais aussi auprès des communautés, particulièrement des femmes et des jeunes pour stimuler la demande. Par ailleurs, il est ressorti des conclusions de ces foras que, bien que la CU soit un produit autorisé et intégré dans le Programme National de PF, sa déclassification en tant que produit du tableau A (nécessitant une prescription médicale) et son autorisation dans le cadre des soins post-viol/inceste devraient être des questions qui mériteraient d'être discutées avec les décideurs et les parlementaires. Aussi, la nécessité d'adapter l'environnement politico-juridique lié à l'offre de services SR avait été fortement soulevée lors de ces réunions stratégiques.

Le Population Council, dans le cadre des efforts de promotion de l'agenda international de la CU, a initié une Journée de Dialogue sur la CU au Sénégal. Cette journée a été financée dans le cadre d'un partenariat entre le Population Council et le Consortium International sur la Contraception 
d'Urgence (ICEC). Cette journée avait pour but de susciter un nouvel engagement autour de la CU chez les autorités publiques et les autres parties prenantes..

\section{CADRE DE LA JOURNEE}

La journée de dialogue sur la CU s'est tenue le 11 juin 2013 à l'hôtel Le Ndiambour à Dakar. Elle a été organisée par le Population Council et le Consortium International sur la Contraception d'Urgence (ICEC) en partenariat avec le Ministère de la Femme, de l'Enfant et de l'Entreprenariat Féminin, le Ministère de la Santé et de l'Action Sociale et le Ministère de la jeunesse, de l'Emploi et de la promotion des Valeurs Civiques.3

\section{OBJECTIFS DE LA JOURNEE DE DIALOGUE}

\section{Objectif général}

L'objectif général était de développer un plan d'actions pour la création d'un environnement politique et juridique plus favorable à l'offre de CU, et pour la vulgarisation de la CU auprès des communautés.

\section{Objectifs spécifiques}

Les objectifs spécifiques de la journée étaient de :

- Procéder à la mise à jour des connaissances sur la contraception d'urgence, les politiques et programmes existants au Sénégal ;

- Partager les résultats de recherche sur la CU faite par le Population Council et d'autres institutions ;

- Partager les expériences des institutions d'encadrement et de prise en charge des jeunes et des femmes en matière de santé sexuelle et reproductive et droits humains ;

- Identifier les contraintes sur le plan politico-juridique et proposer des solutions ;

- Informer le personnel de santé et les communautés sur la CU ;

- Développer un plan d'actions et discuter de sa mise en œuvre.

\section{Methodologie}

La journée a été organisée en séances en plénières et en travaux de groupe.

Les travaux de groupe ont porté sur les trois thématiques suivantes :

- Plaidoyer pour une amélioration de l'environnement politique et juridique pour une meilleure accessibilité des services de CU;

- Vulgarisation de la CU et création de la demande ;

- Offre de services de CU et intégration de la CU dans les structures formelles et non formelles.

La facilitation a été assurée par le Dr Manuel Pina, gynécologue/obstétricien privé. 


\section{DEROULEMENT DE LA JOURNEE}

\section{Cérémonie d'ouverture}

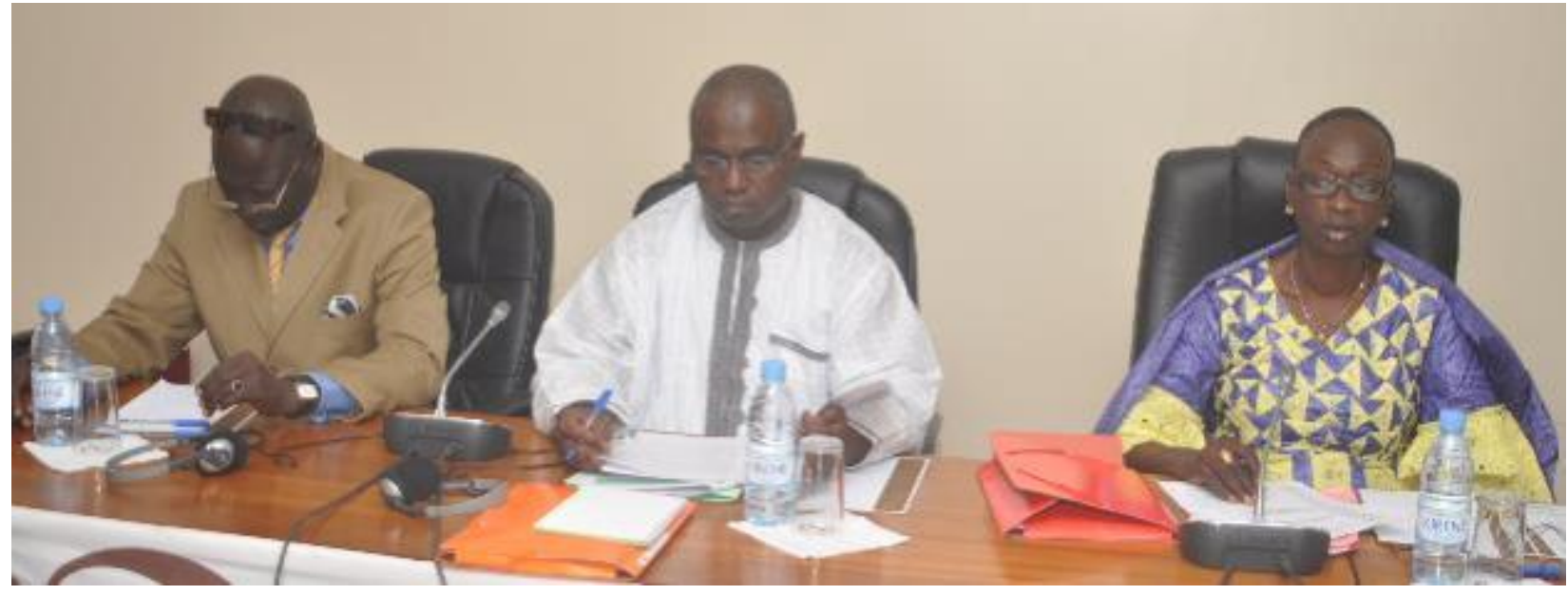

La Cérémonie d'ouverture a été présidée par la Directrice de la Famille, représentant Madame le Ministre de la Femme, de l'Enfant et de l'Entreprenariat Féminin. Cinq allocutions ont été prononcées respectivement par les représentants du Ministère de la Jeunesse, de l'Emploi et de la promotion des Valeurs Civiques, du Ministère de la Santé et de l'Action Sociale, de la représentante d'ICEC, de la Directrice du Population Council au Sénégal et de la représentante du Ministère de la Femme, de l'Enfant et de l'Entreprenariat Féminin.

- Mr Ibrahima Samba Mboup, représentant le Ministère de la Jeunesse, de l'Emploi et de la Promotion des Valeurs Civiques a axé son intervention sur l'intérêt que porte son Ministère à la CU au regard de la forte exposition des adolescents/jeunes aux GND. Il a, à cet effet, rappelé les initiatives menées par le Projet Promotion des Jeunes (PPJ) dans le domaine de la formation des prestataires des Centres Conseils Adolescents (CCA) en vue de renforcer l'offre de CU aux adolescents/jeunes. Le représentant du Ministère a lancé un appel aux partenaires, en particulier au Population Council, pour renforcer la collaboration en faveur de la promotion et de la consolidation de la CU dans les structures d'encadrement des adolescents/jeunes.

- Le Directeur de la DSRSE, Dr Bocar Daff, représentant le Ministre de la Santé et de l'Action Sociale a remercié les partenaires qui ont répondu à l'invitation et salué la présence de la représentante de l'Assemblée Nationale qui est une institution centrale dans la prise de décisions politiques en matière de SR. Dr Daff a ensuite focalisé son intervention, l'importance de la PF dans la prévention des risques sanitaires liés à la grossesse et dans la réduction de la mortalité maternelle et infantile. Il s'est appesanti sur les acquis, notamment, l'intégration de la CU dans le Programme National de SR. Selon le Directeur de la DSRSE, la CU occupe une place centrale dans la prévention des GND et leurs corollaires. Toutefois, bien que la CU soit autorisée, sa vulgarisation auprès des populations constitue un défi majeur à relever. Par ailleurs, il importe de revoir l'environnement juridique pour une déclassification des produits de la CU du tableau A qui impose au plan réglementaire la nécessité d'une prescription médicale. Le Directeur de la DSRSE a conclu ses propos en rappelant que la CU n'est pas une méthode 
régulière de contraception mais sa vulgarisation peut contribuer à l'atteinte des Objectifs 4 et 5 du Millénaire pour le Développement (OMD).

- La Représentante d'ICEC, Mme Sarah Rich, a remercié le Gouvernement du Sénégal et le Population Council d'avoir associé le Consortium à cette importante journée. Elle a salué ensuite la présence des hautes autorités publiques et surtout la présence d'une représentante de l'assemblée nationale à cette réunion. Elle a indiqué que, bien que la question de la CU soit controversée, celle-ci demeure un volet complémentaire des programmes de PF car étant la seule méthode efficace pour prévenir les GND en cas de viol, d'échec d'utilisation de la contraception ou d'absence d'utilisation de la PF. La représente d'ICEC a indiqué, en outre, qu'après avoir réalisé l'étude sur «les Croyances, Attitudes et Pratiques des leaders d'Opinions et des prestataires sur la Contraception d'Urgence », le Population Council, en partenariat avec le Consortium, va engager avec les parties prenantes locales un débat sur la place de la CU et sur la stratégie à développer pour renforcer cette place dans les programmes de PF et assurer la vulgarisation de ce produit auprès des femmes pour qu'elles puissent y accéder quand elles en ont besoin.

- Le Dr Nafissatou Diop, Directrice du Population Council, a remercié les différents Ministères de leur franche collaboration qui a permis la réalisation de cette journée. Elle a mis l'accent sur l'importance des recherches sur la CU et leur dissémination pour améliorer les environnements politique et programmatique pour une meilleure utilisation de ce produit..

- Mme Coumba Ngom Thiam, Directrice de la Famille, et représentante du Ministère de la Femme, de l'Enfant et de l'Entreprenariat Féminin a magnifié la vision de Monsieur le Président de la République du Sénégal qui a motivé l'inscription de l'amélioration de l'accès à des soins de qualité pour les populations en général et pour les femmes en particulier parmi les axes prioritaires de la politique nationale de développement. Elle a rappelé l'importance de la PF dans la stratégie de réduction de la mortalité maternelle et dans l'atteinte des OMD en matière de santé auxquels le Gouvernement du Sénégal a souscrit. Selon la Directrice, l'organisation de cette journée constitue une opportunité pour mutualiser les expériences des différents acteurs pour la promotion de la CU. L'élaboration du Plan d'Action devra servir, selon la Directrice de la Famille, de document de référence pour consolider cette synergie. Elle a terminé son discours en lançant un appel à tous les acteurs pour une mobilisation permanente en faveur de la promotion de la PF et en assurant les participants que les conclusions de la journée seront prises en compte dans les priorités de son Ministère.

Après la cérémonie d'ouverture, plusieurs présentations ont été faites. 


\section{Présentations}

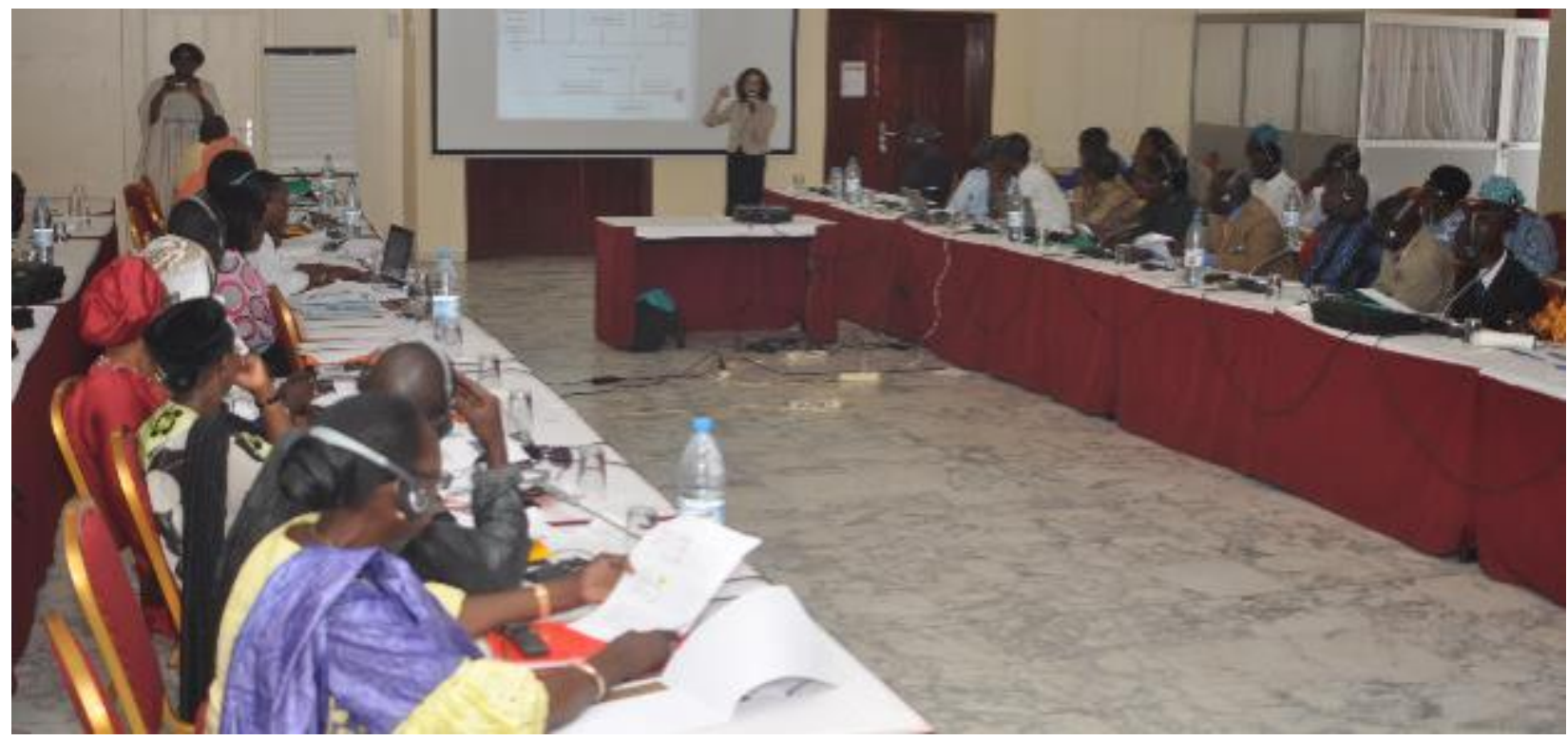

En introduction, Mr Babacar Mané, chargé de programme au Population Council, a situé le contexte de la journée de dialogue sur la CU dans le cadre d'une collaboration entre ICEC et le Population Council pour la promotion de la CU. Cela porte notamment sur : i) la dissémination des résultats de la recherche sur "les croyances, attitudes et pratiques des leaders d'opinions clé et des prestataires sur la CU" auprès des acteurs et dans des revues internationales; ii) l'utilisation des résultats pour le plaidoyer auprès des décideurs; iii) la tenue d'une journée de dialogue avec les parties prenantes. Mr Mané a, en conclusion, rappelé aux participants les objectifs de la journée qui étaient de :

- Partager les résultats de recherche avec les parties prenantes au plus haut niveau ;

- Procéder à une mise à jour sur les dernières informations sur la CU ;

- Susciter un engagement politique et programmatique en faveur de la CU.

A la suite de l'exposé de Mr Mané, Mme Sarah Rich a procédé à un aperçu global de la CU à travers le monde. Les principaux points abordés dans sa présentation ont été les suivants :

- Qu'est-ce que l'ICEC?

- Qu'est-ce que la Contraception d’Urgence ? Et pourquoi est-elle importante?

- Comment les pilules de CU fonctionnent-elles?

- Quelle est la différence entre la CU et l'avortement ?

- La Contraception d’Urgence est-elle sûre?

- L'utilisation répétée de la CU est-elle sûre/appropriée?

- Les adolescents et les jeunes doivent-ils avoir accès à la CU ?

- La CU conduit-elle à des comportements sexuels à risques ? 
- Quels sont les succès, demi-succès, défis en cours ?

Les points de discussion ont porté sur:

- La prise répétée, abusive de la CU ;

- L'accès à la CU ;

- Les effets secondaires ;

- Les effets de la CU sur le cycle menstruel ;

- L'âge auquel on peut prescrire la CU;

- La CU et les adolescents ;

- La CU et les IST/SIDA ;

- L'exposition des adolescents aux IST ;

- La lutte contre les GND ;

- L'insuffisance de l'information sur la CU;

- Comment booster la connaissance en mettant l'accent sur le marketing social ;

- Le degré de satisfaction des utilisatrices ;

- La CU et les droits humains ;

- La CU par rapport aux autres méthodes de PF ; concurrence ou non?

- La classification de la CU au tableau A.

En réponse aux questions soulevées, la représentante d'ICEC a :

- Confirmé l'absence d'effets secondaires à long terme liés à l'utilisation de la CU ;

- Réaffirmé, que "l'utilisation répétée ne pose pas de risques connus pour la santé" et "que son utilisation est sûre même lorsque les femmes la prennent plus d'une fois dans un même cycle menstruel";

- Insisté sur la nécessité d'informer les femmes que l'utilisation d'une méthode régulière et continue est recommandée comme étant la façon la plus efficace de prévenir une grossesse non désirée ;

- Conclu en mentionnant que les femmes doivent avoir toutes les informations sur toutes les méthodes, cependant, celles ayant des rapports sexuels non protégés devraient être orientées vers la CU;

- Les femmes doivent être informées sur l'efficacité sans omettre de leur dire que la CU ne protège pas contre les IST.

Le Dr Salisu Ishaku, Gestionnaire de Programmes au Population Council-Nigéria, a ensuite fait une présentation sur l'expérience du Nigéria intitulée «le rôle potentiel de la Contraception d'Urgence (CU) dans le Plan National de Développement du Nigéria : Discussions Emergentes». Selon le présentateur, malgré l'importance de l'accès à la PF y compris la CU dans la Politique Nationale de Population du Nigéria, l'offre de la CU est exclusivement assurée par le secteur privé contrairement aux autres méthodes de PF. Ceci entraîne une irrégularité dans l'approvisionnement et conduit à un manque de confiance dans les produits disponibles. Dr Ishaku a rappelé que, même si la plupart de gens l'ignorent, il y a 
l'existence de produits contrefaits de PF, notamment concernant les pilules de CU. Le présentateur s'est aussi appesanti sur les points de vue émergents tels que : i) l'inclusion des produits de la CU dans la liste nationale des médicaments essentiels; ii) l'utilisation des champions de la planification familiale au niveau communautaire pour promouvoir l'accès à la CU à toutes les catégories de femmes; iii) la mise en œuvre d'activités de plaidoyer intensif basé sur des évidences scientifiques pour dissiper les idées erronées sur la CU; iv) la conception d'approches novatrices pour mettre à la disposition des prestataires de services, des clients potentiels et des décideurs des informations exactes.

Les points de discussion soulevés ont porté sur:

- L'absence de structures gouvernementales qui offrent la CU;

- L'utilisation de la CU par les femmes mariées ;

- La durée d'action de la CU;

- Les cibles potentielles.

En guise de réponse, Dr Salisu Ishaku a :

- Rappelé le caractère légal de la CU et souligné que, même si le gouvernement ne s’implique pas, il appuie de manière indirecte la promotion de la CU.

A la suite de la présentation du Dr Ishaku, le Dr Chimère Diaw, Chef de la Division Planification familiale à la DSRSE exposé la place de la CU dans le Programme de PF du Sénégal, notamment son rôle dans la réduction des Besoins Non Satisfaits (BNS) en matière de PF et par ricochet la baisse des grossesses à risque, de la mortalité maternelle et infantile. Il a insisté sur le fait que la CU est une méthode d'appoint qui ne peut remplacer les méthodes régulières de PF. De plus, il a fait l'historique de l'introduction de la CU au Sénégal qui a démarré en 1998 avec son inclusion dans le curriculum de formation des prestataires en PF et sa mention dans les PNP qui précisent que les pilules classiques et le DIU étaient utilisables pour la CU. Le Dr Diaw a par la suite passé en revue les différentes recherches menées au Sénégal par les acteurs et partenaires du Ministère de la Santé et de l'Action Sociale sur la CU et le développement des supports IEC. Il a, en outre, abordé les perceptions/opinions sur la CU notamment son appréciation mitigée qui entrave sa vulgarisation. Il a aussi mentionné que la CU demeure encore peu connue et que son utilisation reste faible malgré que le produit soit disponible en vente libre dans les officines privées. Il a conclu sur une note de Thoraya Obaid (ancienne Directrice Exécutive de l'UNFPA) qui indiquait que le principal défi à relever est que : "le monde doit sauver les femmes, pour que les femmes sauvent le monde..."

Les points de discussion ont porté sur:

- Le rôle des religieux ;

- L'absence de soutien conjugal quant au recours des femmes à la PF;

- La position des religieux quant à l'utilisation répétée de la CU, notamment à l'occasion des cérémonies religieuses;

- L'insuffisance d'études sociologiques sur la CU;

- L'intégration de la CU dans la Stratégie Nationale de Développement Economique et Social du Sénégal ; 
- La nécessité de réduire les coûts de la CU ;

- La position du MSAS par rapport à l'utilisation répétée de la CU ;

- Les différents produits de $\mathrm{CU}$ disponibles au niveau des officines privées à des prix abordables (ex : le Norvel 72 qui coute 1225 FCFA);

- L'âge d'éligibilité parce qu'il y a des jeunes de 13-14 ans victimes de viol.

Dans ses réponses, le Dr Chimère Diaw a précisé que :

- $\quad \mathrm{La} C \mathrm{CU}$ n'est pas une méthode régulière ;

- Il faudra se rapprocher des universitaires pour voir s'il existe des études sociologiques sur la CU.

A la suite du Dr Diaw, Mr Babacar Mané a procédé au partage des résultats des recherches menées sur la CU au Sénégal. Il a mentionné le faible niveau de connaissance et d'utilisation de la CU qui sont des constats récurrents que l'on retrouve dans la plupart des études. En outre, selon le présentateur, bien que l'environnement politique et programmatique soit favorable à l'offre de CU, la persistance d'opinions défavorables et d'idées erronées constituent des contraintes majeures à son utilisation. Il a conclu en insistant sur l'importance de mener des actions de plaidoyer en faveur de la CU en vue de réduire les BNS en PF et d'adresser certaines questions telles que les violences basées sur le genre (viols, incestes) et leurs corollaires (avortements, infanticides).

Les principales interpellations ont porté sur :

- Le respect de la législation en vigueur;

- Ne pas faire la promotion d'une seule méthode au détriment des autres déjà disponibles ;

- La CU et les droits humains ;

- La CU et les cas de viols publics et conjugaux ;

- L'âge minimum pour l'utilisation de la CU. 


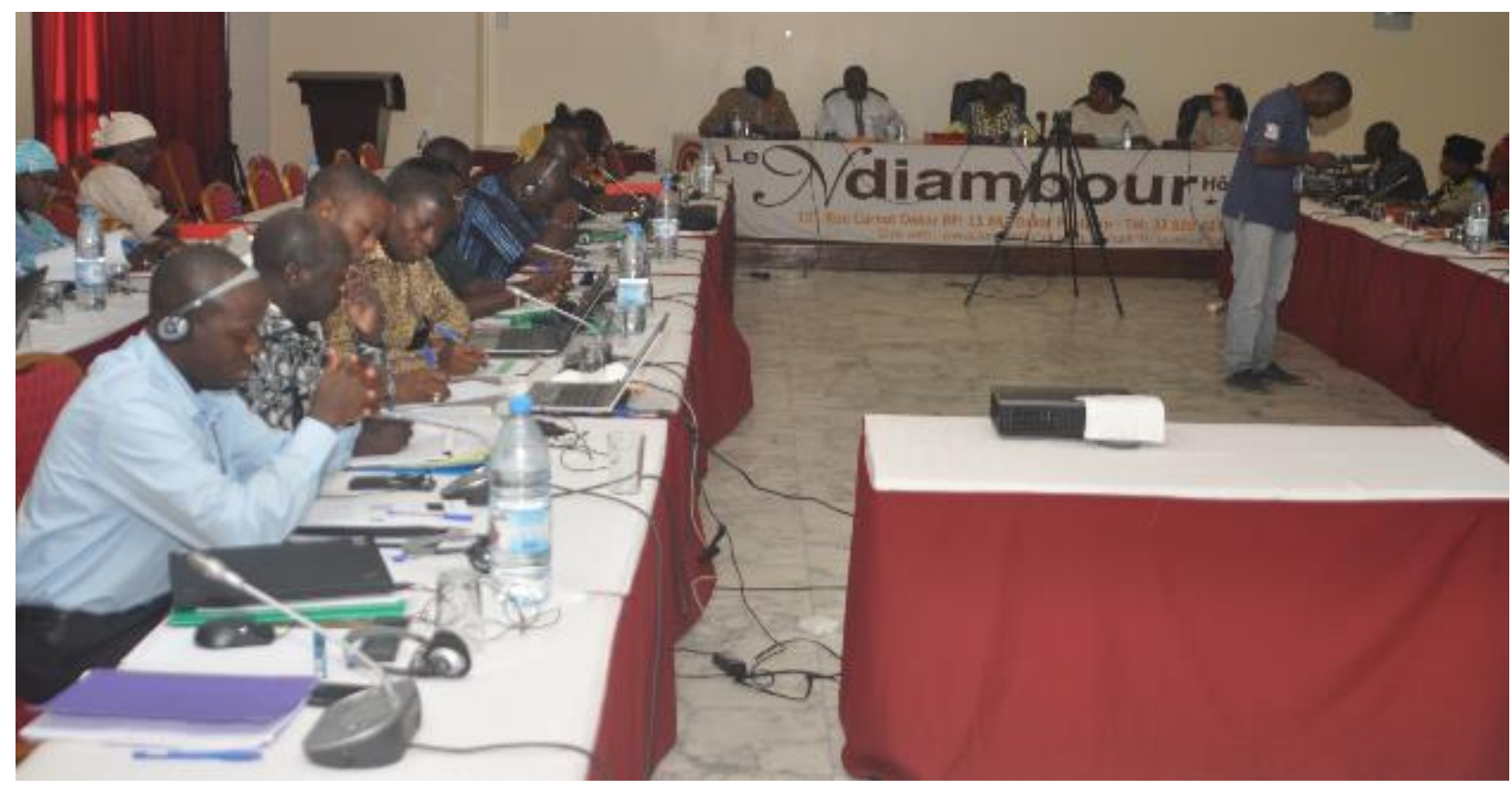

A la suite des présentations générales, certains acteurs clés ont été invités à partager leur expérience en matière de CU.

La représentante de la Maison d'Arrêt et de Correction du Camp Pénal (Prison des femmes) a indiqué que les infanticides et les avortements provoqués occupent une part non négligeable parmi les motifs de détention chez leurs pensionnaires qui sont issues pour la plupart de milieu pauvre et sont analphabètes. Elle a mentionné la difficulté d'organiser des séances de causeries sur la PF au niveau de la prison. La représentante du Camp pénal a insisté sur l'importance que la CU pourrait avoir sur la stratégie de prévention des infanticides et la réinsertion sociale des femmes détenues.

La Directrice de la Famille a, quant à elle, décliné les deux missions principales de son ministère. Elle a noté le besoin indéniable d'une bonne vulgarisation de la CU qui permettrait de faire baisser le nombre d'infanticides et aiderait les femmes à y recourir pour se protéger car sociologiquement la femme ne doit pas refuser l'acte sexuel à son mari. La présentatrice a indiqué que la CU entre parfaitement dans le programme de lutte contre les avortements clandestins et les infanticides et elle doit figurer dans le paquet de soins post-viol qui est un sujet important qui touche directement la famille. Elle a confirmé l'engagement de son Ministère à tout mettre en œuvre pour sa vulgarisation lors d'événements spéciaux tels que la quinzaine de la femme, la journée de la femme. Pour ce faire, les associations de femmes et les Organisations Communautaires de Base (OCB) pourraient être mises à contribution. Les approches familiales et celles intégrant les violences basées sur le genre seront utilisées.

Pour le représentant du Projet Promotion des Jeunes (PPJ), son institution est arrivée à renverser la tendance en permettant aux jeunes d'accéder aux services de PF. Il a souligné l'appui de l'UNFPA qui a permis de former tous les agents des Centres Conseils Adolescents (CCA) à la CU. Selon le représentant du Ministère de la Jeunesse, la CU est actuellement intégrée dans les activités de 
routine des CCA. Il a toutefois noté le faible taux d'utilisation de la CU malgré la gratuité du produit au niveau de leurs structures.

Le représentant de l'ASBEF a partagé l'expérience de son institution dans la promotion de la CU. Il a insisté sur les acquis en termes de renforcement des capacités des prestataires et des relais. La contribution de l'ASBEF s'est par ailleurs manifestée à travers la réalisation d'études et l'intégration de la CU dans les stratégies de prise en charge des cas de viols et d'abus sexuels qui a été déterminante dans la promotion de la CU. Il a terminé par un récapitulatif de l'offre de CU dans les cliniques de l'ASBEF depuis 2009.

\section{Travaux de groupe}

A la suite des séances plénières, trois (3) groupes de travail ont été constitués pour l'élaboration du Plan d'Action. Il s'agit de :

- Plaidoyer pour une amélioration de l'environnement politique et juridique pour une meilleure accessibilité des services de CU;

- Vulgarisation de la CU et création de la demande ;

- Offre de services de CU et intégration de la CU dans les structures formelles et non formelles.

Au niveau de chaque groupe un modérateur et un rapporteur ont été désignés.

Les contributions des différents groupes sont déclinées à travers l'identification des lignes d'actions prioritaires.ci-dessous :

\section{Groupe 1 : Plaidoyer pour une amélioration de l'environnement politique et judiciaire pour une meilleure accessibilité des services de CU}

Les actions prioritaires suivantes ont été proposées :

- Développer des activités de plaidoyer en direction des parlementaires pour rendre effective la loi sur la SR adoptée depuis 2005. Le RSJ et le Comité de Lutte contre les Violences Faites aux Femmes devront assurer le lead pour cette acticité ;

- Développer des actions en vue de réviser et assouplir les dispositions réglementaires relatives à l'offre des produits PF. Les cibles

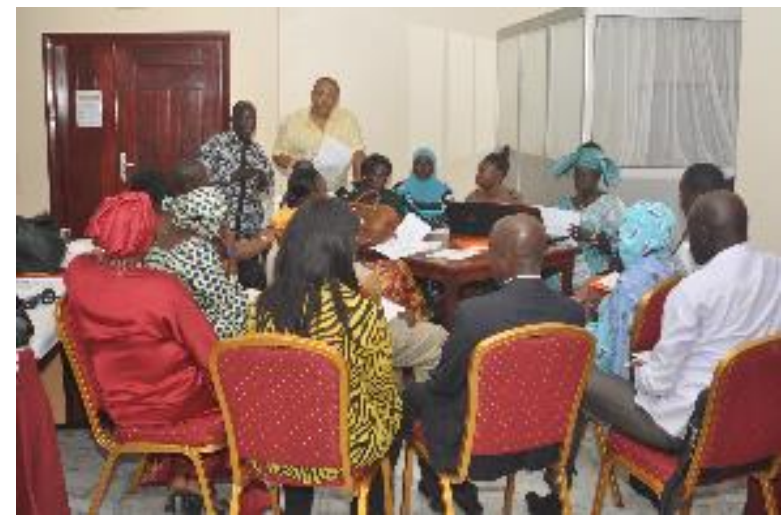
prioritaires sont la Direction de la pharmacie, le Syndicat des pharmaciens, l'Ordre des pharmaciens ;

- Développer un partenariat et des mécanismes d'intégration pour rendre disponibles dans les officines privées les produits PF des services publics. La cellule Partenariat Public-Privé devra jouer un rôle clé dans ce processus ; 
- Réviser les politiques, Normes et protocoles de PF pour la prise en compte des femmes en situation de vulnérabilité (femmes en zones de conflit).

\section{Groupe 2 : Vulgarisation de la CU et création de la demande}

Les travaux du groupe 2 ont permis d'identifier les problèmes liés à la demande et de proposer les solutions pour lever ces contraintes :

- Quelles sont les barrières liées à la demande/utilisation de la CU?

o Méconnaissances de la CU aussi bien par les prestataires que par les populations ;

o Inaccessibilité aux services de contraception d'urgence liée à :

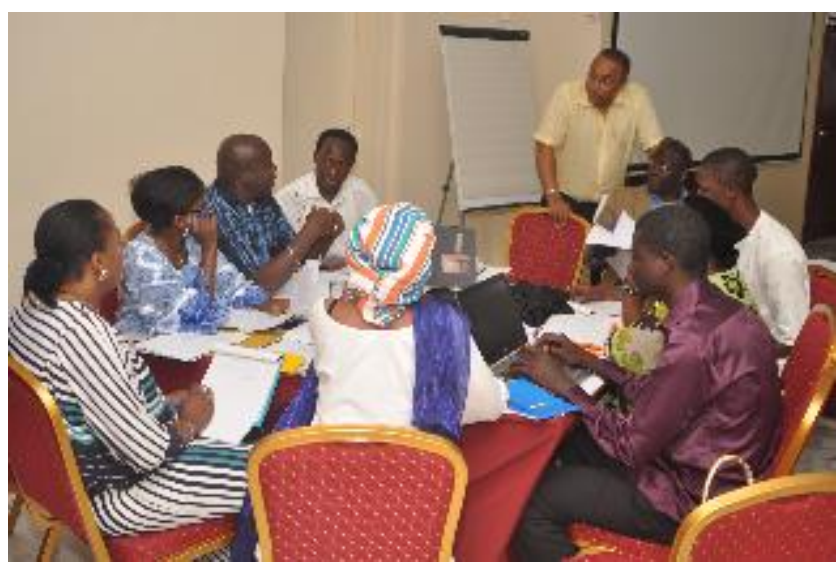

- Comportement des prestataires ;

- Organisation des services (horaires, services offerts, temps d'attente) ;

o Barrières socioculturelles :

- Tabous ;

- Religion ;

- Ignorance ;

- Analphabétisme.

- Quels sont les groupes qui ont le plus besoin des informations sur les services de CU ?

o Les adolescents/jeunes;

o Les religieux;

o Les couples;

o Les leaders communautaires ;

o Les femmes célibataires.

- Quelle est la stratégie/approche de communication la plus appropriée ? et pour quelle cible?

o Argumentaires pour les religieux ;

o Communication de masse ;

o Causeries;

o Communication interpersonnelle.

- Quels sont les canaux/supports?

o Mass media;

o Pairs éducateurs;

o IEC intra-muros;

o Activités IEC au niveau communautaire; 
o NTIC (mobile, internet, réseaux sociaux etc.).

\section{Recommandations}

- Rendre disponible le produit ;

- Renforcer la supervision des points de prestations de services ;

- Renforcer la collaboration multisectorielle ;

- Sécuriser la distribution de la CU.

\section{Groupe 3 : Offre de services de CU et intégration de la CU dans les structures formelles et non formelles}

Les principales interventions proposées ont porté sur :

- La formation des acteurs à la base, notamment: des agents des services du Développement communautaire, des CPRS, du GEEP, des établissements pénitenciers, de la Croix Rouge, de la FAWE, de l'ONCAV, des services du Ministère de la jeunesse, du CENAF et des CEDAF, et du Ministère de la formation professionnelle ;

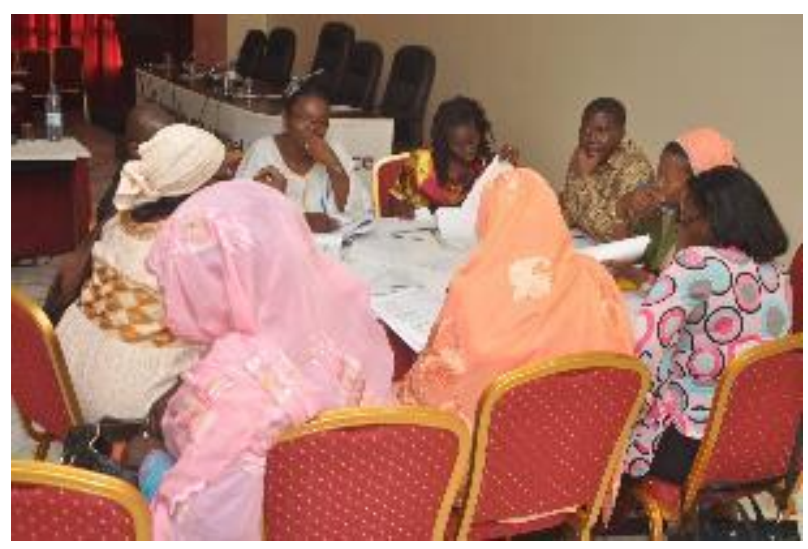

- L'introduction par le Ministère de l'Education Nationale d'un module dans le curriculum de formation des élèves/étudiants sur la prévention des GND incluant l’information sur la CU.

- La mise en œuvre d'activités d'IEC ciblant les femmes et les hommes, les jeunes filles et les garçons, les professeurs, les relais techniques, les populations carcérales, les femmes vulnérables, le personnel féminin de l'Armée ;

- La mise en œuvre d'activités de plaidoyer auprès des leaders d'opinions, notamment: les leaders religieux, les leaders politiques, les parlementaires, les élus locaux et les leaders coutumiers ;

- La mise en place de campagnes de communication ciblant les populations, notamment à travers les radios communautaires.

\section{Déclaration conjointe d'engagement des trois Ministères partenaires}

Suite aux travaux de groupes, un draft de déclaration conjointe d'engagement du Ministère de la Femme, de l'Enfant et de l'Entreprenariat Féminin, du Ministère de la Santé et de l'Action Sociale, et du Ministère de la jeunesse, de l'Emploi et de la promotion des Valeurs Civiques a été partagé avec les participants pour l'opérationnalisation d'un plan d'action pour l'amélioration de l'accès aux services de Contraception d'Urgence (CU). 
Au cours des discussions, il a été suggéré d'inclure d'autres parties prenantes, telles que le Ministère de la Justice, le Ministère de l'Education Nationale, la Société Civile, parmi les signataires. Cependant au vu de l'objectif d'assurer l'application du plan, il a été reconnu que les 3 Ministères partenaires étaient les signataires les plus appropriés. Des éditions ont été suggérées. Le Population Council se chargera de finaliser la déclaration en vue de sa signature officielle.

\section{Clôture de la journée de partage}

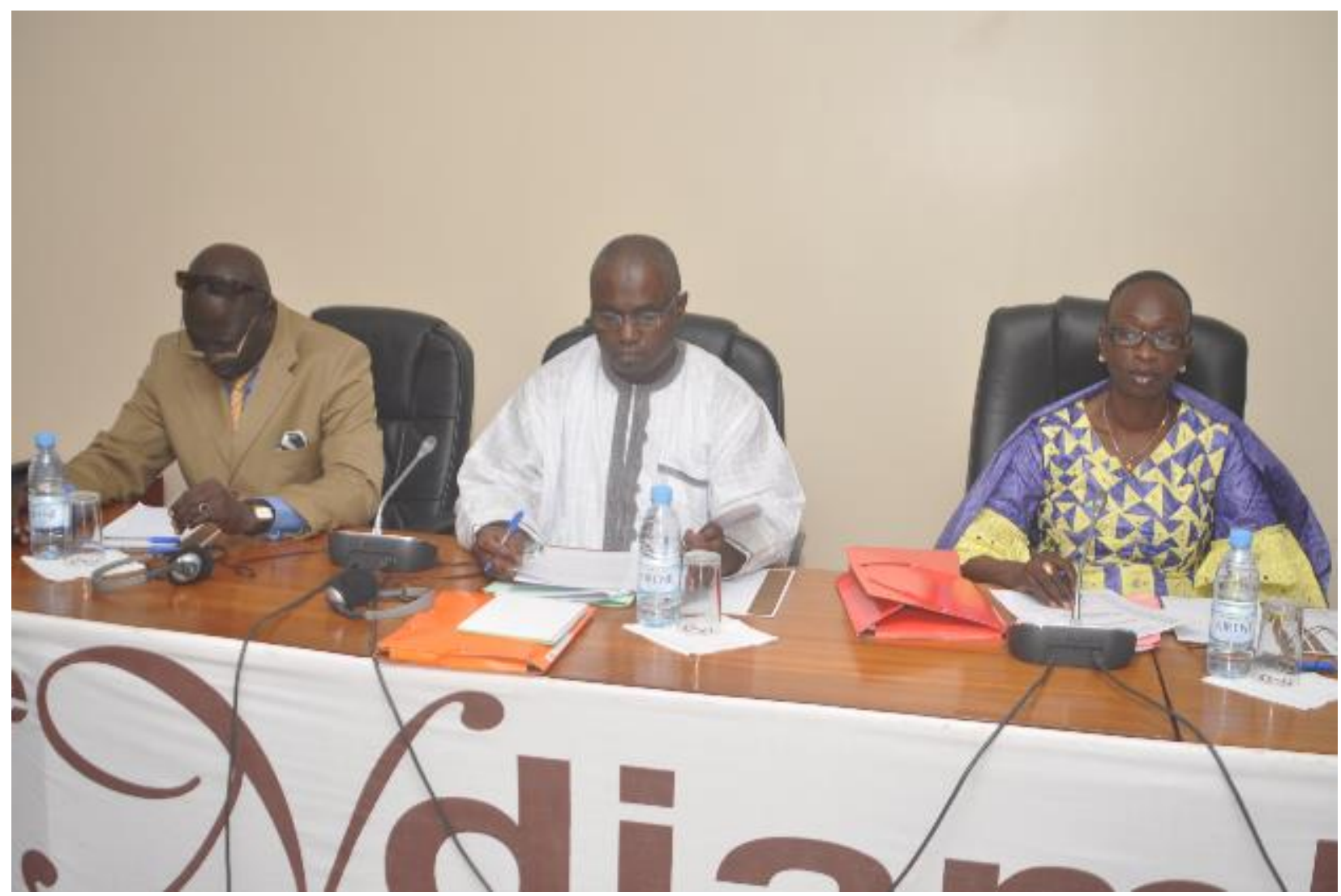

La cérémonie de clôture a été présidée par le Dr Bocar Daff, Directeur de la DSRSE. Dans son allocution, le Dr Daff a rappelé le rôle du parlement dans l'adoption des lois, d'où l'importance de sensibiliser les parlementaires sur le sujet. Il a également insisté sur la nécessité d'intégrer la CU dans le cadre global de la PF. Le Dr Daff a conclu son intervention en remerciant les participants pour leurs contributions aux travaux et a salué l'engagement du Population Council aux côtés du Ministère de la Santé et de l'Action Sociale. 


\section{ANNEXES}

\section{Annexe 1 : Agenda}

\section{JOURNEE DE DIALOGUE SUR LA CONTRACEPTION D'URGENCE}

DATE : 11 JUIN 2013. LIEU (HOTEL LE NDIAMDOUR)

\section{AGENDA}

$8: 30-9: 00$

9:00 - 9:15

9:15 - 9:30

9:30 - 9:45

$9: 45-10: 00$

$10: 00-10: 15$

$10: 15-10: 30$

$10: 30-11: 15$

$11: 15-13: 00$

$13: 00-14: 30$

$14: 30$ - $15: 30$

$15: 30-16: 30$

$16: 30-17: 00$

$17: 00$

\section{Cérémonie d'ouverture}

Allocution du Population Council

Allocution de I'ICEC

Allocution du représentant du Ministre de la Jeunesse, de l'Emploi et de la Promotion des Valeurs civiques

Allocution du représentant du Ministre de la Santé et de l'Action Sociale

Allocution de Mme le Ministre de la Femme, de l'Enfance et de l'Entreprenariat Féminin

Contexte de l'étude et de la journée de dialogue (Population Council Sénégal)

Présentation de la CU : mode d'action et aperçu de la situation à travers le monde (ICEC)

Pause café

Expérience du Nigeria en matière de contraception d'urgence (Population Council-Nigéria)

- $\quad$ La CU dans le programme de PF du Nigéria

- Expérience de la Journée de Dialogue du Nigéria

Expérience du Sénégal en matière de contraception d'urgence (DSRSE)

- $\quad$ La CU dans le programme de PF du Sénégal

Expériences des acteurs locaux. (DF, PPJ, Camp pénal, ASBEF)

Résultats des recherches sur la CU au Sénégal (population en générale, pharmacies, KOL). (Population Council Sénégal)

Travaux de groupe. Objectif : Développer un plan d'action pour la vulgarisation de la CU auprès de communauté (contraintes, solutions/actions à mener, échéancier, responsables).

3 axes d'intervention :

- $\quad$ Plaidoyer pour une amélioration de l'environnement politique et juridique pour une meilleure accessibilité à la CU.

- Vulgarisation de la CU par les prestataires et les agents de santé/relais communautaires.

- Intégration de l'information sur la CU dans les structures d'encadrement formelles et non formelles des femmes et des jeunes (systèmes d'enseignement, institutions d'insertion et de réinsertion, etc.)

Déjeuner

Travaux de groupe (suite)

Séance plénière (Présentation des rapports des groupes)

Prochaines étapes

Comment mettre en place le plan d'action

Cérémonie de clôture par Mme le Ministre de la Santé et de l'Action Sociale

Déclaration conjointe d'engagement du Ministère de la femme, de l'enfance et de l'entreprenariat féminin, du Ministère de la Santé et de l'Action Sociale, et du Ministère de la Jeunesse, de l'Emploi et de la Promotion des Valeurs civiques pour opérationnaliser le plan d'action

\section{(2) Population Council}




\section{Annexe 2 : Travaux de groupe pour développer un plan d'action pour la vulgarisation de la $\mathrm{CU}$ auprès de communauté.}

Le produit des trois groupes de travail est présenté ci-dessous :

Groupe 1: Plaidoyer pour une amélioration de l'environnement politique et judiciaire pour une meilleure accessibilité des services de CU

\begin{tabular}{|c|c|c|c|}
\hline PLAN D'ACTION POUR AI & IELIORER L'ACCES A & ERVICES DE CU & \\
\hline Intervention : Plaidoyer pour & une amélioration de l'env & ement politique et ju & \\
\hline Activité no & Cibles & Partenaires/ & Resnonsables \\
\hline & & acteurs & \\
\hline Plaidoyer pour l'effectivité de & & & RSJ \\
\hline la Loi SR & Parlementaires & & CLVF \\
\hline & Syndicat & & \\
\hline Revision de la reglementation & Direction Pharmacie & & \\
\hline & Ordre des pharmaciens & & \\
\hline & MSAS & & \\
\hline Partenariat public-privé & $\begin{array}{l}\text { Cellule partenariat } \\
\text { public/privé }\end{array}$ & & Direction des EPS \\
\hline Révision des politiques et & MSAS & & $\mathrm{RS} \mathrm{J} / \mathrm{AJS}$ \\
\hline normes $^{1}$ & MFEEF & & CLVF \\
\hline Intégration des produits $\mathrm{PF}$ & MSAS & & \\
\hline $\begin{array}{l}\text { du public au niveau des } \\
\text { officines privées }\end{array}$ & Direction pharmacie & Population Council & DSRE \\
\hline
\end{tabular}

${ }^{1}$ : Révision des politiques et normes pour la prise en compte des femmes extrêmement vulnérables (femmes en conflit)

\section{Groupe 2: Vulgarisation de la CU et création de la demande}

- Quelles sont les barrières liées à la demande/utilisation de la CU ?

- Méconnaissances de la CU aussi bien par les prestataires que par la population

o Inaccessibilité aux services de contraception d'urgence

- Comportement des prestataires

- Organisation des services (horaires, services offerts, temps d'attente)

o Barrières socioculturelles

- Tabous

- Religion

- Ignorance

- Analphabétisme 
- Quels sont les groupes qui ont le plus besoin des informations sur les services de CU?

o Les adolescents/jeunes

o Les religieux

o Les couples

o Les leaders communautaires

o Les femmes célibataires

- Quels types d'informations sont les plus appropriées pour atteindre et informer les cibles?

- Quelle est la stratégie/approche de communication la plus appropriée ? et pour quelle cible?

o Argumentaires pour les religieux

o Communication de masse

o Causeries

o Communication interpersonnelle

- Quels sont les canaux/supports?

o Mass media

o Educateurs pairs

o IEC intra muros

o Activités IEC au niveau communautaire

o NTIC (mobile, internet, réseaux sociaux etc....)

\section{Recommandations spécifiques}

- Rendre disponible le produit

- Renforcer la supervision des PPS

- Renforcer la collaboration multisectorielle

- Sécuriser la distribution de la CU

\begin{tabular}{|l|l|l|l|}
\hline \multicolumn{2}{|l|}{ PLAN D'ACTION POUR AMELIORER L'ACCES AUX SERVICES DE CU } \\
\hline \multicolumn{2}{|l|}{ Intervention : Vulgarisation de la CU et création de la demande } \\
\hline Activité $\mathrm{n}^{\circ} \ldots$. & Cibles & Partenaires/acteurs & Responsables \\
\hline Former les prestataires sur la CU & $\begin{array}{l}\text { Personnel qualifié et } \\
\text { ASC/Matrones et Pairs } \\
\text { éducateurs }\end{array}$ & $\begin{array}{l}\text { DSRSE } \\
\text { PTF }\end{array}$ & $\begin{array}{l}\text { DSRSE } \\
\text { Partenaires }\end{array}$ \\
\hline $\begin{array}{l}\text { Réorganiser les points de prestations } \\
\text { de services }\end{array}$ & Tous les PPS & MSAS et Partenaires & DSRSE \\
\hline $\begin{array}{l}\text { Elaborer un plan de communication } \\
\text { pour la CU }\end{array}$ & $\begin{array}{l}\text { MSAS (SNIEPS, } \\
\text { DSRSE) et Partenaires }\end{array}$ & SNEIPS \\
\hline
\end{tabular}




\section{Groupe 3 : Offre de services de CU et intégration de la CU dans les structures formelles et}

non formelles

\begin{tabular}{|c|c|c|c|}
\hline \multicolumn{4}{|c|}{$\begin{array}{l}\text { PLAN D'ACTION POUR AMELIORER L'ACCES AUX SERVICES DE CU } \\
\text { Intervention } n^{\circ} 1 \text { : élargissement de l'offre de service en CU }\end{array}$} \\
\hline Activité $n^{\circ} \ldots$. & Cibles & $\begin{array}{l}\text { Partenaires/ } \\
\text { acteurs }\end{array}$ & Responsables \\
\hline $\begin{array}{l}\text { - Former les } \\
\text { acteurs à la base }\end{array}$ & $\begin{array}{l}\text { CEDAF } \\
\text { Développement communautaire } \\
\text { CPRS } \\
\text { GEEP } \\
\text { Etablissement pénitentiaire } \\
\text { Croix Rouge } \\
\text { FAWE } \\
\text { ONCAV } \\
\text { Ministère de la jeunesse } \\
\text { Ministère de la formation } \\
\text { professionnelle } \\
\text { Ministère de l'Education Nationale }\end{array}$ & $\begin{array}{l}\text { Ministère de la santé } \\
\text { Ministère de la } \\
\text { jeunesse } \\
\text { ONG } \\
\text { Partenaires } \\
\text { techniques et } \\
\text { financiers } \\
\text { associations }\end{array}$ & DSRSE \\
\hline $\begin{array}{l}\text { - IEC } \\
\text { (information, } \\
\text { Education, } \\
\text { communication) }\end{array}$ & $\begin{array}{l}\text { Les femmes et les hommes } \\
\text { Les jeunes filles et garçons } \\
\text { Les Adultes } \\
\text { Professeurs relais techniques } \\
\text { Population carcérale } \\
\text { Les femmes vulnérables } \\
\text { Le personnel féminin armé }\end{array}$ & & $\begin{array}{l}\text { Les services } \\
\text { techniques et les } \\
\text { structures } \\
\text { d'encadrement non } \\
\text { formelles }\end{array}$ \\
\hline $\begin{array}{l}\text { - Campagnes de } \\
\text { plaidoyer au } \\
\text { niveau national }\end{array}$ & $\begin{array}{l}\text { Leaders d'opinions } \\
\text { Leaders religieux } \\
\text { Leaders politiques } \\
\text { les parlementaires } \\
\text { les ONGs } \\
\text { les élus locaux } \\
\text { les leaders coutumiers }\end{array}$ & & $\begin{array}{l}\text { Les services } \\
\text { techniques et les } \\
\text { structures } \\
\text { d'encadrement non } \\
\text { formelles }\end{array}$ \\
\hline $\begin{array}{l}\text { - Campagnes de } \\
\text { communication } \\
\text { médias }\end{array}$ & $\begin{array}{l}\text { La population } \\
\text { Union des Radios Communautaires }\end{array}$ & & $\begin{array}{l}\text { Ministère de la santé } \\
\text { et le Ministère de la } \\
\text { communication }\end{array}$ \\
\hline
\end{tabular}

* L'équipe de Population Council se chargera de la finalisation du plan d'action. 


\section{Annexe 3 : Draft - Déclaration conjointe d'engagement}

Déclaration conjointe d'engagement du Ministère de la Femme, de l'Enfant et de 1'Entreprenariat Féminin, du Ministère de la Santé et de l'Action Sociale, et du Ministère de la Jeunesse, de l'Emploi et de la Promotion des Valeurs Civiques pour opérationnaliser le plan d'action pour l'amélioration de l'accès aux services de Contraception d'Urgence (CU).

L'accès rapide, facile et à un prix abordable à la Contraception d'Urgence (CU) dans les 120 heures qui suivent un rapport sexuel non protégé peut réduire de façon significative les taux de grossesses non désirées et d'avortements. Des études ont démontré que l'accès à la CU pouvait réduire de $43 \%$ les avortements. L'OMS, reconnaissant que l'utilisation de la CU était sans risque pour la femme, avait fortement recommandé aux Etats membres de promouvoir l'accès aux services de CU et de l'intégrer dans les programmes nationaux. Toutefois, malgré l'adoption de cette méthode dans les programmes nationaux et une plus grande disponibilité des pilules contraceptives d'urgence spécifiques, l'accès demeure toujours très limité pour la grande majorité des femmes qui en ont besoin. La méconnaissance du produit par les populations, d'une part, l'attitude des prestataires et leurs perceptions souvent négatives sur le produit, d'autre part, constituent des facteurs qui contribuent à limiter l'accès des femmes aux services de CU. Par ailleurs, l'environnement juridique peu clair par rapport à cette question constitue une contrainte supplémentaire.

Le Forum national sur la CU qui s'était tenu à Dakar en Octobre 2010 avait fortement recommandé une vulgarisation du produit au niveau des communautés.

C'est ainsi que le Ministère de la Femme, de l'Enfant et de l'Entreprenariat Féminin, le Ministère de la Santé et de l'Action Sociale, le Ministère de la Jeunesse, de l'Emploi et de la Promotion des Valeurs Civiques,

- Considérant, l'impact négatif que les grossesses non désirées et les avortements peuvent avoir sur le bien-être des femmes et l'avenir des jeunes filles

- Considérant l'engagement des pouvoirs publics dans la politique de prévention de la morbidité et de la mortalité maternelle et de l'amélioration du bien-être des adolescents/jeunes;

- Considérant les droits des populations à un accès équitable à l'information et à des services de santé de qualité garantis par la constitution,

- Considérant l'engagement du Gouvernement à promouvoir l'accès à des services de planification familiale

- Considérant l'intérêt de développer une approche multisectorielle pour garantir toutes les chances de succès dans les interventions

Ont décidé de conjuguer leurs efforts pour inscrire dans leurs programmes respectifs les actions pour améliorer l'accès aux services de CU. A cet effet, nous nous engageons à mettre en œuvre les stratégies et activités proposées dans le plan d'action pour la promotion de la $\mathrm{CU}$, ceci dans nos domaines respectifs de compétence.

Fait à Dakar, le 11 juin 2013.

\section{Le Représentant du MSAS}

\author{
Le Représentant \\ du MJEVC
}

\section{Le Représentant du MFEEF}




\section{Annexe 4 : Liste des participants}

\begin{tabular}{|c|c|c|}
\hline $\mathbf{N}^{\circ}$ & Prénoms et Nom & Structure \\
\hline 1 & Mme Coumba Ngom Thiam & Direction de la Famille \\
\hline 2 & Ndeye Khourédia Diop & Direction de la Famille \\
\hline 3 & Mme Cissé Astou Diop & Direction de la Famille \\
\hline 4 & Alioune Thiam & Direction de la Famille \\
\hline 5 & Mme Marième Dieng & Direction Equité et Genre \\
\hline 6 & Ndeye Fatou B. Sarr & $\begin{array}{l}\text { Centre Départemental d'Assistance et } \\
\text { de Formation pour la Femme (CEDAF }\end{array}$ \\
\hline 7 & Fatoumata Diamanka & $\begin{array}{l}\text { Centre Départemental d'Assistance et } \\
\text { de Formation pour la Femme (CEDAF }\end{array}$ \\
\hline 8 & Lamine Diop & $\begin{array}{l}\text { Centre National d'Assistance et de } \\
\text { Formation pour la Femme (CENAF }\end{array}$ \\
\hline 9 & Dr Bocar Daff & $\begin{array}{l}\text { Direction de la Santé de la } \\
\text { Reproduction et de la Survie de } \\
\text { l'Enfant (DSRSE) }\end{array}$ \\
\hline 10 & Marie Jésus Bouabé & $\begin{array}{l}\text { Direction de la Santé de la } \\
\text { Reproduction et de la Survie de } \\
\text { l'Enfant (DSRSE) }\end{array}$ \\
\hline 11 & Dr Papa Chimère Diaw & $\begin{array}{l}\text { Direction de la Santé de la } \\
\text { Reproduction et de la Survie de } \\
\text { l'Enfant (DSRSE) }\end{array}$ \\
\hline 12 & Mbagnick Diouf & $\begin{array}{l}\text { Service National de l'Education et de } \\
\text { l'Information Sanitaire (SNEIPS) }\end{array}$ \\
\hline 13 & Adama Cissé & $\begin{array}{l}\text { Service National de l'Education et de } \\
\text { l'Information Sanitaire (SNEIPS) }\end{array}$ \\
\hline 14 & Gora Niang & $\begin{array}{l}\text { Service National de l'Education et de } \\
\text { l'Information Sanitaire (SNEIPS) }\end{array}$ \\
\hline 15 & Ibrahima Samba Mboup & Projet Promotion des Jeunes (PPJ) \\
\hline 16 & Mbaké Diouf & Centre Conseil Adolescent (CCA) \\
\hline 17 & Madou Cissé & Centre Conseil Adolescent (CCA) \\
\hline 18 & Djibril Sadio & Centre Conseil Adolescent (CCA) \\
\hline 19 & Laouratou Baldé & $\begin{array}{l}\text { Direction des Centres d'Enseignement } \\
\text { Technique Féminin }\end{array}$ \\
\hline 20 & Dr Mame Mbayame Dione & $\begin{array}{l}\text { Commission Santé de l'Assemblée } \\
\text { Nationale }\end{array}$ \\
\hline 21 & Hamath Anne & Ville de Guédiawaye \\
\hline 22 & $\begin{array}{l}\text { Ndeye Sokhna Ndiaye } \\
\text { Marone }\end{array}$ & Centre de Guidance Infantile \\
\hline 23 & Adama Sanokho & $\begin{array}{l}\text { Association des Sages-femmes d'Etat } \\
\text { du Sénégal }\end{array}$ \\
\hline 24 & Dr Fatou Ndiaye Dème & Ordre des Pharmaciens \\
\hline 25 & Ndèye Dagué Gueye & Association des Femmes Handicapées \\
\hline
\end{tabular}




\begin{tabular}{|c|c|c|}
\hline $\mathbf{N}^{\circ}$ & Prénoms et Nom & Structure \\
\hline & & du Sénégal \\
\hline 26 & Ndeye Ndiaye & Cam Pénal (Prison des femmes) \\
\hline 27 & Papa Makhéte Djité & $\begin{array}{l}\text { Organisme National des Activités de } \\
\text { Vacances (ONCAV) }\end{array}$ \\
\hline 28 & Athie Binta D. Sarr & $\begin{array}{l}\text { Action pour le Développement } \\
\text { (ACDEV) }\end{array}$ \\
\hline 29 & Soukeye Ndao Diallo & $\begin{array}{l}\text { Association des femmes juristes } \\
\text { Sénégalaises(AJS) }\end{array}$ \\
\hline 30 & Abdou Aziz Mandiang & $\begin{array}{l}\text { Association Sénégalaise pour le Bien- } \\
\text { être Familial (ASBEF) }\end{array}$ \\
\hline 31 & Ndèye Maguette Diop & Childfund \\
\hline 32 & Penda Seck Diouf & $\begin{array}{l}\text { Comité de Lutte contre les Violences } \\
\text { faites aux Femmes }\end{array}$ \\
\hline 33 & Nicolas Mendy & Croix Rouge Sénégalaise \\
\hline 34 & Aminata Traoré Seck & Division du Contrôle Médical Scolaire \\
\hline 35 & Serigne Moussa Diop & DOEF \\
\hline 36 & $\begin{array}{l}\text { Adama Ndeye Carine } \\
\text { Mbengue }\end{array}$ & $\begin{array}{l}\text { Forum for African Women } \\
\text { Educationalist (FAWE) }\end{array}$ \\
\hline 37 & Dr Siga Diop & FHI360 \\
\hline 38 & Dié Marie Faye & $\begin{array}{l}\text { Groupe pour l'Education et } \\
\text { l'Enseignement de la Population } \\
(\text { GEEP) }\end{array}$ \\
\hline 39 & Moussa Gueye & IntraHealth \\
\hline 40 & Bineta Diop & IPAS \\
\hline 41 & Thiaba Sembene & Réseau Siggil Jiggen (RSJ) \\
\hline 42 & Eugène Kaly & Le Soleil \\
\hline 43 & Dr Manuel Pina & $\begin{array}{l}\text { Médecin privé - Modérateur de la } \\
\text { journée }\end{array}$ \\
\hline 44 & Sarah Rich & $\begin{array}{l}\text { International Consortium for } \\
\text { Emergency Contraception }\end{array}$ \\
\hline 45 & Edouard Yao & Indépendant \\
\hline 46 & Nafissatou Diop & Population Council \\
\hline 47 & Salisu Ishaku & Population Council \\
\hline 48 & Babacar Mané & Population Council \\
\hline 49 & Ababacar Thiam & Population Council, consultant \\
\hline
\end{tabular}

